\title{
ALANINE BLENDS FOR ESR MEASUREMENTS OF THERMAL NEUTRON FLUENCE IN A MIXED RADIATION FIELD
}

\author{
M. Marrale1,3,*, M. Brai ${ }^{1,3}$, G. Gennaro ${ }^{1}$, A. Triolo ${ }^{1}$, A. Bartolotta ${ }^{2,3}$, M.C. D’Oca ${ }^{2}$ and G. Rosi ${ }^{4}$ \\ ${ }^{1}$ Dipartimento di Fisica e Tecnologie Relative, Università di Palermo, Viale delle Scienze, Edificio 18, 90128 \\ Palermo, Italy \\ ${ }^{2}$ Dipartimento Farmacochimico Tossicologico e Biologico, Università di Palermo, Via Archirafi 32, 90123 \\ Palermo, Italy \\ ${ }^{3}$ Unità CNISM, Palermo and Gruppo V Sezione INFN, Catania, Italy \\ ${ }^{4}$ Centro di Ricerche ENEA, Casaccia, Roma, Italy
}

\begin{abstract}
In this paper, the results of a study on the electron spin resonance (ESR) dosimetry to measure thermal neutron fluence in a mixed radiation field (neutron and photons) are presented. The ESR responses of alanine dosemeters with different additives are compared. In particular, the ${ }^{10} \mathrm{~B}$-acid boric and the $\mathrm{Gd}$-oxide were chosen to enhance the sensitivity of alanine dosemeters to thermal neutrons. Irradiations were carried out inside the thermal column of the TAPIRO reactor of the ENEA center, Casaccia Rome. The main results are a greater neutron sensitivity and a smaller lowest detectable fluence for the dosemeters with gadolinium than for dosemeters of alanine with ${ }^{10} \mathrm{~B}$, which is well known to be much more sensitive to thermal neutrons than simple alanine.
\end{abstract}

\section{INTRODUCTION}

In the cancer treatment named neutron capture therapy (NCT), the tumour cells are hit through a binary process characterised by the delivery to tumour cells of a compound carrying nuclei with an high thermal neutron capture cross section, and by the irradiation of the tumour volume with thermal neutrons. Actually, in the radiotherapic treatments, epithermal neutrons are used that thermalise at depths of a few centimetres in tissue, maximising the dose into the tumour volume. To optimise the procedure and to enhance the effects on the tumour cells, an opportune quantity of the target nuclei inside and in the neighbourhood of the tumour cells and an adequate fluence of thermal neutrons to tumour cells are needed. Thus, two variables need to be controlled in NCT: the concentration of high cross section nuclei in the tumour and the thermal neutron fluence.

Moreover, a nucleus must have two fundamental properties to be used for NCT: it must show a high neutron capture cross section and the emitted particles after nuclear reaction must have high LET to release all their energy in the neighbourhoods of the cell target. The nuclei that usually are used in NCT are ${ }^{10} \mathrm{~B}$ and $\mathrm{Gd}^{(1-3)}$.

The ${ }^{10} \mathrm{~B}$ offers an high capture cross section to thermal neutron (3838-barn). The reaction induced by thermal neutron in the ${ }^{10} \mathrm{~B}$ is ${ }^{10} \mathrm{~B}(\mathrm{n}, \alpha){ }^{7} \mathrm{Li}$. In the detail, two parallel nuclear fission processes occur after the absorption of a thermalised neutron.

*Corresponding author: marrale@difter.unipa.it
The excited ${ }^{11} \mathrm{~B}$ nucleus splits producing two high LET ions, ${ }_{2}^{4} \mathrm{He}$ (alpha particles) and ${ }_{3}^{7} \mathrm{Li}$. The paths of these two high-LET particles are of the order of $(4-9 \mu \mathrm{m})$. Further, a gamma photon $(2.31 \mathrm{MeV}$ with probability of $94 \%$ and $2.79 \mathrm{MeV}$ with probability $6 \%$ ) is emitted ${ }^{(2)}$.

The gadolinium is used because two of the seven stable gadolinium isotopes have high neutron capture cross section, ${ }^{155} \mathrm{Gd}$ (75 000 barn) and ${ }^{157} \mathrm{Gd}$ (250 000 barn). The atomic fractions of ${ }^{155} \mathrm{Gd}$ and ${ }^{157} \mathrm{Gd}$ are 0.148 and 0.156 , respectively; therefore, the effective neutron capture cross section is 50 000 barn. When a neutron is captured by the gadolinium, a complex reaction is started. In this reaction, complex inner shell transitions generate prompt $\gamma$-emission displacing an inner-core electron, which involves the emission of an internalconversion electron emission, and finally the Auger electron emission, together with soft X-ray and photon emissions. In this case, the high LET particles are the Auger electron, which release their energy in several nanometers ${ }^{(2)}$.

Thermal neutron fluences are currently measured with a technique using bare and cadmium-covered gold foils ${ }^{(4)}$.

Alanine electron spin resonance (ESR) dosimetry is an accurate method for photon and charged particle beams, useful both in radiotherapy and in the industrial application of ionising radiation. An ESR dosimetry with alanine for thermal neutron beams is not sensitive enough because of the low neutron capture cross section of the nuclei in the alanine molecule. Consequently, the interaction of thermal neutrons with the nuclei is not much probable, and 


\section{MARRALE ETAL.}

so the amount of energy deposited, and the number of radicals produced in alanine are very small.

To improve the neutron interactions probability, a nucleus with a high thermal neutron capture cross section could be added into the alanine dosemeters to act as a neutron capture centre. The compound already investigated to improve the ESR dosemeter sensitivity is the $\mathrm{B}(\mathrm{OH})_{3}^{(5-7)}$. A few efforts have been made to develop some alternative compounds to measure fluence with ESR dosemeter without the use of alanine ${ }^{(8)}$. This and also other investigators then take advantage of the ${ }^{6} \mathrm{Li}$ content in natural or enriched $\mathrm{Li}$; ${ }^{6} \mathrm{Li}$ has a high neutron capture cross section. This method is used for thermoluminescence as well as ESR- dosemeters used for neutron and photon mixed field dosimetry at $\mathrm{BNCT}^{(9)}$.

In this paper, a method to enhance the radical yield and, consequently, the ESR signal amplitude response of alanine to thermal neutrons by mixing gadolinium oxide and boric acid with alanine is presented. The method is based on the fact that when a mixture of alanine with ${ }^{10} \mathrm{~B}$ or $\mathrm{Gd}$ is irradiated with thermal neutrons, the stable isotopes, ${ }^{10} \mathrm{~B}$ or $\mathrm{Gd}$, may experience a neutron capture reaction with an high probability. The projectiles from the nuclear reaction could impinge on the alanine molecules, producing extra free radicals. The problem with this method is that a number of different interactions occur that cause creations of different radicals in different amounts in alanine with the result of difficulties in controlling the signal intensity.

\section{MATERIALS AND METHODS}

\section{ESR dosimetry}

An ESR alanine-based dosimetry is a wellestablished method of gamma, electron and proton dose measurement ${ }^{(10)}$.

Solid-state pellets for an ESR dosimetry were made using a blend of $\mathrm{L}-\alpha$-alanine (Fluka, Buchs, Switzerland), gadolinium-oxide (Aldrich Chem. Co.), boric acid (Aldrich Chem. Co.) in known proportions by weight.

The pellets were realised following a procedure previously optimised ${ }^{(11)}$ by pressing a blend of $94 \%$ of the appropriate active material [either $\mathrm{B}(\mathrm{OH})_{3}-$ alanine or $\mathrm{Gd}_{2} \mathrm{O}_{3}$-alanine or alanine alone], $5 \%$ of polyethylene (Polysciences, $\mathrm{MW}=700$ ) as binder and $1 \%$ of magnesium stearate (Carlo Erba, Milano, Italy) as lubricant. The pellets of alanine with $\mathrm{Gd}_{2} \mathrm{O}_{3}$ addition were realised by pressing a blend where the two constituent alanine and $\mathrm{Gd}_{2} \mathrm{O}_{3}$ were present in equal proportion in weight $(47 \%)$, analogously for the pellets of alanine with $\mathrm{B}(\mathrm{OH})_{3}$.

Pellets of $\sim 4 \mathrm{~mm}$ in diameter and 2.5, 2.5 and $1.9 \mathrm{~mm}$ in thickness for alanine, $\mathrm{B}(\mathrm{OH})_{3}$-alanine and $\mathrm{Gd}_{2} \mathrm{O}_{3}$-alanine, respectively, were obtained with an effective $Z / A$ ratio of $0.503,0.503$ and 0.417 , respectively.

The ESR spectra were recorded at room temperature with a Bruker ECS 106 spectrometer operating at $9.7 \mathrm{GHz}$. The peak-to-peak intensity of the main line of the spectrum was chosen as the dosedependent quantity.

The following ESR recording parameters were chosen to obtain the highest signal-to-noise ratio: central field, $348.5 \mathrm{mT}$; sweep width, $20 \mathrm{mT}$, microwave power, $4 \mathrm{~mW}$; modulation amplitude, $1 \mathrm{mT}$; time constant, $655 \mathrm{~ms}$ and sweep time, $42 \mathrm{~s}$.

The alanine, $\mathrm{B}(\mathrm{OH})_{3}$-alanine and $\mathrm{Gd}_{2} \mathrm{O}_{3}-$ alanine dosemeters were calibrated using a ${ }^{60} \mathrm{Co}$ source (Alcyon II, General Electric, France) used for radiotherapy treatments at the Radiotherapy Department of the Oncology Hospital 'M. Ascoli' in Palermo. Dosemeters irradiations were performed inside a Perspex phantom $\left(30 \times 30 \times 30 \mathrm{~cm}^{3}\right)$ at the water equivalent depth of $5 \mathrm{~cm}$; the field size was $15 \times 15 \mathrm{~cm}^{2}$, and the source-to-dosemeter distance was $80 \mathrm{~cm}$; The dose rate at the effective dosemeter location was evaluated with an overall uncertainty of $2 \%(95 \%$ confidence level) using the ENEA (Ente per le Nuove tecnologie, l'Energia e l'Ambiente, Italy) secondary standard ionisation chamber.

The absorbed dose in dosemeters with $\mathrm{Gd}_{2} \mathrm{O}_{3}$ is different from dose in alanine or $\mathrm{B}(\mathrm{OH})_{3}$-alanine because of the large differences in mass energy absorption properties. However, the aim of the calibration of the dosemeters to ${ }^{60} \mathrm{Co}$ photons is to correlate the ESR response of each blend with wellestablished values of dose in water. The $\gamma$-photon calibration is used to obtain an estimate of the ESR signal due to the $\gamma$-component in the mixed field.

\section{Thermal neutron irradiations}

Thermal neutron irradiations have been performed at the TAPIRO reactor, at the ENEA Casaccia Center, near Rome. Cylindrical dosemeter holders (22 $\mathrm{mm}$ diameter, $7 \mathrm{~mm}$ height) were made with Teflon and utilised for irradiations. Four dosemeters were placed, in suitable small cavities, inside the holders, and irradiated inside the thermal column of the TAPIRO nuclear reactor at thermal neutron fluence in the range between $\sim 10^{12}$ and $\sim 5$ $10^{13} \mathrm{n} \mathrm{cm}^{-2}$ measured through the activation gold foils. Neutron flux in thermal column is mainly composed of thermal neutrons ( $90 \%$ of the total). The epithermal neutrons are the principal component of the rest of the neutron flux ( $\sim 9 \%$ of the total).

\section{RESULTS AND DISCUSSION}

Table 1 shows, for each value of used thermal neutron fluences (from $2.4 \times 10^{12}$ up to $3.92 \times$ $10^{13} \mathrm{n}_{\mathrm{th}} \mathrm{cm}^{-2}$ ), the tissue neutron kerma calculated 
Table 1. Thermal neutron fluences used to irradiate the dosemeters; for each fluence the tissue neutron kerma was calculated using the appropriate kerma factor; the gamma dose was measured through an ionisation chamber.

\begin{tabular}{lcc}
\hline $\begin{array}{l}\text { Fluence } \\
\left(10^{12} \mathrm{n}_{\mathrm{th}} \mathrm{cm}^{-2}\right)\end{array}$ & $\begin{array}{c}\text { Tissue neutron } \\
\text { kerma (Gy) }\end{array}$ & $\begin{array}{c}\text { Gamma } \\
\text { dose }(\mathrm{Gy})\end{array}$ \\
\hline 2.65 & 0.740 & 0.205 \\
3.92 & 1.09 & 0.325 \\
21.2 & 5.92 & 1.64 \\
39.2 & 10.9 & 3.25 \\
\hline
\end{tabular}

using the kerma factors ${ }^{(12)}$, and the gamma dose measured by ENEA laboratories. The ratio between neutron kerma and gamma dose was $\sim 3.5$ in each irradiation.

The irradiated dosemeters of alanine and alanine with boric acid show the well-known ESR spectrum centred at about $g=2$. The dosemeters of $\mathrm{Gd}_{2} \mathrm{O}_{3}-$ alanine show also the ESR signal due to $\mathrm{Gd}_{2} \mathrm{O}_{3}$, which is very wide $(\sim 270 \mathrm{mT})$ and centred in the $g=2$ region field. The contribution of $\mathrm{Gd}_{2} \mathrm{O}_{3}$ signal in the magnetic field range, wherein ESR spectrum of alanine is present, is linear. To eliminate this background signal, a linear baseline was subtracted to each spectrum of $\mathrm{Gd}_{2} \mathrm{O}_{3}$-alanine. Figure 1 shows the ESR spectrum of $\mathrm{Gd}_{2} \mathrm{O}_{3}$-alanine (a) before and (b) after the baseline elimination. The peak-to-peak amplitude for these dosemeters was measured after this baseline elimination. The spectra of the three blends exposed to gamma photons and to neutrons are shown in Figure 2. The various spectra of the blends exposed to different quality radiation are

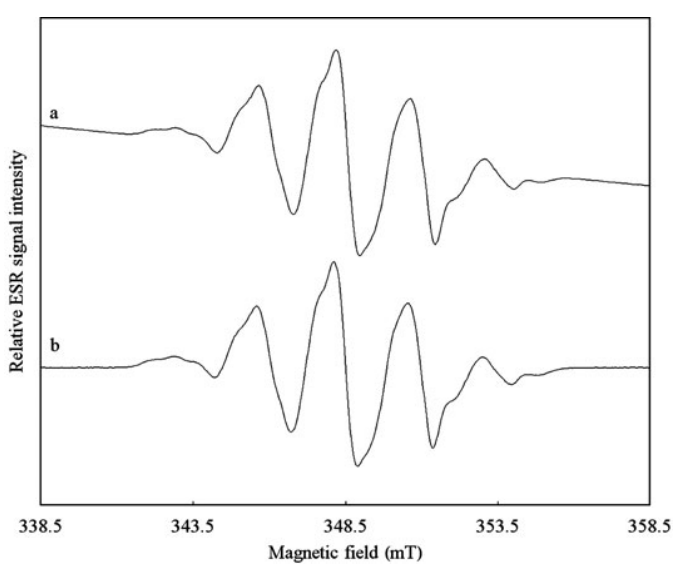

Figure 1. ESR signal of a dosemeter of $\mathrm{Gd}_{2} \mathrm{O}_{3}$-alanine exposed to the mixed field (neutron fluence $3.92 \times 10^{13}$ ) (a) with the ESR signal of gadolinium and (b) after the subtraction of this signal. similar. However, a detailed analysis of the radicals that contribute to the ESR signal is behind the aim of this work.

For each fluence and for each dosemeter type, the measured total ESR signal $H$ was due to both neutrons and gamma photons; for each irradiation condition, at least three dosemeters were used. The average value of $H$ was evaluated; the corresponding standard deviation was found to decrease from $\sim 10$ to $2 \%$ with increasing fluence.

The gamma dose was converted to the corresponding ESR signal intensity $H_{\gamma}$ using the ${ }^{60} \mathrm{Co}$ calibration function of ESR dosemeters. The difference of the total ESR signal and gamma ESR signal $\left(H_{\mathrm{n}}=H-H_{\gamma}\right)$ is the signal attributable to thermal neutrons.

These experimental data of the alanine and $\mathrm{B}(\mathrm{OH})_{3}$-alanine were analysed through a linear fit

$$
H_{n}=a+b \Phi
$$

where $\Phi$ represents the fluence values provided by the irradiation centre and $b$ is the sensitivity of the blend to neutrons.

In the experimental data of $\mathrm{Gd}_{2} \mathrm{O}_{3}$-alanine, a slight saturation behaviour was observed; this behaviour can be due to an high radical concentration, which involves saturation. Therefore, the data were

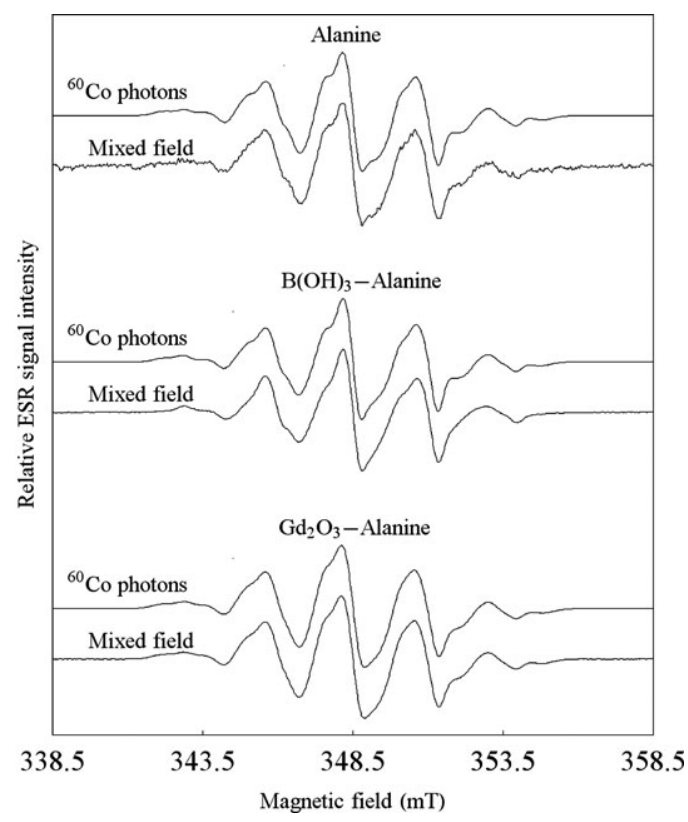

Figure 2. Comparison of the ESR spectra of alanine, $\mathrm{B}(\mathrm{OH})_{3}$-alanine and $\mathrm{Gd}_{2} \mathrm{O}_{3}$-alanine exposed to gamma photons and to mixed field. Each spectrum is normalised to its own maximum value. 
M. MARRALE ET AL.

Table 2. Results of the fitting procedure for the three blends of samples. In the last column the LMF computed as discussed in the main text is reported.

\begin{tabular}{|c|c|c|c|c|c|}
\hline \multirow[t]{2}{*}{ Blend } & \multicolumn{3}{|c|}{ Parameter } & \multirow{2}{*}{$\begin{array}{l}\text { Correlation } \\
\text { coefficient }\end{array}$} & \multirow{2}{*}{$\begin{array}{c}\mathrm{LMF} \\
\left(10^{10} \mathrm{n}_{\mathrm{th}} \mathrm{cm}^{-2}\right)\end{array}$} \\
\hline & $a$ & & & & \\
\hline \multirow[t]{2}{*}{$\begin{array}{l}\text { Alanine } \\
\mathrm{B}(\mathrm{OH})_{3} \text {-alanine }\end{array}$} & $\begin{array}{l}(6 \pm 3) \times 10^{-2} \\
(-2 \pm 4) \times 10^{-1}\end{array}$ & \multicolumn{2}{|c|}{$\begin{array}{c}(3.88 \pm 0.04) \times 10^{-13} \\
(49.1 \pm 0.9) \times 10^{-13}\end{array}$} & $\begin{array}{l}0.9976 \\
0.9968\end{array}$ & $\begin{array}{c}615 \\
46\end{array}$ \\
\hline & $c$ & $d$ & $e$ & & \\
\hline $\mathrm{Gd}_{2} \mathrm{O}_{3}$-alanine & $(33 \pm 5) \times 10^{2}$ & $(5 \pm 5) \times 10^{10}$ & $(20 \pm 3) \times 10^{13}$ & 0.9984 & 5.8 \\
\hline
\end{tabular}

fitted with the following function, which takes into account this saturation trend:

$$
H_{\mathrm{n}}=c\left(1-\mathrm{e}^{-(\Phi+d) / e}\right)
$$

where $c, d$ and $e$ are the parameters of the nonlinear fitting procedure.

In Table 2, the values of the best-fit parameters ( \pm one standard deviation) and the correlation coefficients are reported.

Figure 3 shows the ESR signal $H_{\mathrm{n}}$ as a function of thermal neutron fluence for all blends with alanine. In this figure, the best-fit curves are also shown. Both Figure 3 and Table 2 show that after thermal neutron irradiation, the ESR dosemeters with $\mathrm{B}(\mathrm{OH})_{3}-$ alanine compound showed ESR signals up to $\sim 12$ fold stronger in intensity when compared with the dosemeters with alanine as the sole active component. Moreover, it is evident that the addition of gadolinium in alanine enhances very much the sensitivity (about a factor 35 with respect to pure alanine) of the pellets. The sensitivity of alanine dosemeters with $\mathrm{Gd}_{2} \mathrm{O}_{3}$ is greater (about three times) than that one of $\mathrm{B}(\mathrm{OH})_{3}-$ alanine. These results indicate that, even though the probability of neutron reactions in Gd is about 13 times higher than in ${ }^{10} \mathrm{~B}$, the sensitivity ratio is about three. According to literature ${ }^{(2)}$, after the Gd neutron capture reaction, the Auger electrons and the internal conversion electrons release their energy within a few micrometers. Therefore, these particles release their energy inside the dosemeter, whereas the $\mathrm{X}$ and gamma photons release most of their energy outside the dosemeter. Evidently, even though the number of neutron captured is greater with $\mathrm{Gd}$ addition than ${ }^{10} \mathrm{~B}$ addition, the energy released in the dosemeter by Auger electrons and internal conversion electrons for each Gd neutron capture process is smaller than the energy released by

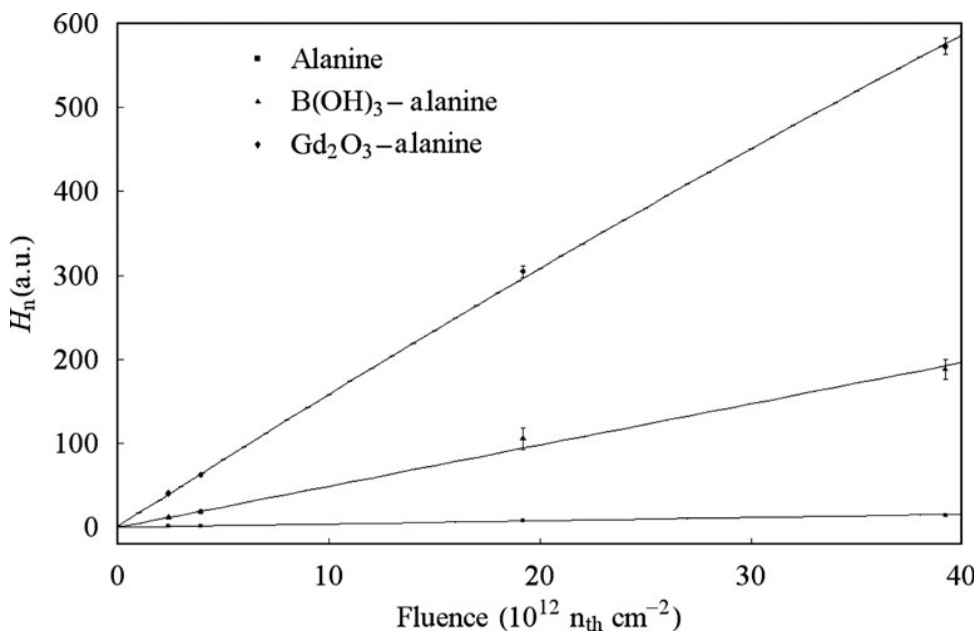

Figure 3. Response curves of alanine, $\mathrm{B}(\mathrm{OH})_{3}$-alanine and $\mathrm{Gd}_{2} \mathrm{O}_{3}$-alanine as a function of the fluence. The fitting curves adopted are explained in the main text. 


\section{NEUTRON DOSIMETRY WITH ALANINE BLENDS}

the alpha particles and lithium ion produced in the ${ }^{10} \mathrm{~B}$ neutron capture. However, the total energy released by secondary particle produced in the $\mathrm{Gd}$ neutron capture is greater than in the ${ }^{10} \mathrm{~B}$ neutron capture; therefore, the number of free radicals is greater with $\mathrm{Gd}$ and the sensitivity to neutron is enhanced.

To verify the improvement related with the addition of gadolinium, an estimation of the lowest measurable fluence (LMF) was made. The LMF was evaluated as the fluence value that produces in the irradiated pellets an ESR signal equal to the mean value of the background in unirradiated pellets plus 10 standard deviations ${ }^{(13)}$. The background signal of six unirradiated pellets for each dosemeter type was measured in the magnetic field range where the ESR signal of the free radicals produced after irradiation is expected to appear (between 347.7 and $349.2 \mathrm{mT}$ ). After a measurement of the background signal and of its standard deviation, the LMF for the alanine and the $\mathrm{B}(\mathrm{OH})_{3}$-alanine dosemeters can be obtained through an inversion of equation (1); in particular, $\mathrm{LMF}=\left[\left(\mathrm{H}_{\mathrm{bg}}+10 \sigma_{\mathrm{bg}}\right)-a\right] / b, \quad$ where $H_{\mathrm{bg}}$ is the background signal and $\sigma_{\mathrm{bg}}$ is its standard deviation. On the other hand, the LMF for the $\mathrm{Gd}_{2} \mathrm{O}_{3}$-alanine dosemeters can be obtained through an inversion of equation (2), in particular, $\mathrm{LMF}=$ $-d-e \times \ln \left[1-\left(H_{\mathrm{bg}}+10 \sigma_{\mathrm{bg}}\right) / c\right]$. The values of the LMF for all alanine blends are reported in the last column of Table 2.The lowest detectable fluence is much smaller in the dosemeters with the gadolinium than in the dosemeters without gadolinium. The $\mathrm{B}(\mathrm{OH})_{3}$-alanine shows a LMF value smaller than simple alanine but, however, greater than that one of $\mathrm{Gd}_{2} \mathrm{O}_{3}$-alanine. The addition of gadolinium therefore significantly enhances the sensitivity and reduces very much the LMF. Both these effects are due to the high neutron capture cross section and to the high LET particles, produced in the nuclear reactions induced by thermal neutrons, which release the energy in the proximity of the Gd nuclei ${ }^{(2)}$.

As final remarks, the addition of gadolinium in alanine/ESR dosemeters described in this paper improves significantly the dosimetric properties of the alanine dosemeters because it increases the sensitivity to the neutron beam and it reduces the lowest detectable fluence. In particular from the comparison carried out in this work, it results evident that the enhancements of sensitivity and the reduction of the LMF with the addition of the gadolinium are better than those observed with the addition of ${ }^{10} \mathrm{~B}$ acid boric. The results show that it is possible to enhance the sensitivity to neutrons of ESR/alanine dosimetry through the gadolinium addition and suggest the applicability of ESR in mixed field of gamma photons and neutrons for typical values of fluence used in NCT.

\section{ACKNOWLEDGEMENTS}

The research described in this paper was supported by a grant from the 'Università di Palermo'. The authors are grateful to V. Caputo of the Radiotherapy Department of the A.R.N.A.S. 'M.Ascoli', Palermo. The authors are grateful to Marcello Mirabello for technical support.

\section{REFERENCES}

1. Barth, R. F., Soloway, A. H. and Fairchild, R. G. Boron neutron capture therapy for cancer. Cancer Res. 50, 1061 (1990).

2. Salt, C., Lennox, A. J., Takagaki, M., Maguire, J. and Hosmanea, S. Boron and gadolinium neutron capture therapy. Russ. Chem. Bull., Int. Ed. 53(9), 1871-1888 (2004).

3. Culbertson, N. and Jevremovic, T. Computational assessment of improved cell-kill by gadolinium-supplemented boron neutron capture therapy. Phys. Med. Biol. 48, 3943-3959 (2003).

4. American Society for Testing and Materials (ASTM). Standard practice for determining neutron fluence rate, fluence, and spectra by radiactivation techniques. ASTM E261-90. Annual Book of ASTM Standards, 12(02), 57 (1990).

5. Urena-Nunez, F., Galindo, S. and Azorin, J. An alanine-boron compound for thermal neutron fluence measurements. Part I: synthesis and development. Appl. Radiat. Isot. 49, 1657-1664 (1998).

6. Urena-Nunez, F., Galindo, S. and Azorin, J. An alanine-boron compound for thermal neutron fluenee measurements. Part 2: EPR response. Appl. Radiat. Isot. 50, 763-767 (1999).

7. Bartolotta, A., D’Oca, M. C., Lo Giudice, B., Brai, M., Borio, R., Forini, N., Salvatori, P. and Manera, S. Combined TL and ${ }^{10} B$-alanine ESR dosimetry for BNCT. Radiat. Prot. Dosim. 110, 627-630 (2004).

8. Herrera, E., Ureña-Núñez, F. and Delfín Loya, A. Lithium carbonate $\left(\mathrm{Li}_{2} \mathrm{CO}_{3}\right)$ as a material for thermal neutron fluence measurements. App. Radiat. Isot. 63, 241-246 (2005).

9. Lund, E., Gustafsson, H., Danilczuk, M., Sastry, M.D. and Lund, A. Compounds of ${ }^{6} \mathrm{Li}$ and natural $\mathrm{Li}$ for EPR dosimetry in photon/neutron mixed radiation fields. Spectrochim. Acta A60, 1319-1326 (2004).

10. American Society for Testing and Materials. Standard practice for use of the alanine-EPR dosimetry system. ASTM 1670-94. 1995 Annual Book of ASTM Standards, 12(02), (Philadelphia, USA: ASTM) (1995).

11. Bartolotta, A., Brai, M., Caputo, V., De Caro, V., Giannola, L.I., Rap, R. and Teri, G. ESR solid state dosimetry: behaviour of various amino-acids and blend preparation procedures. Radiat. Prot. Dosim. 84, 293-296 (1999).

12. International Commission on Radiation Units and Measurement. Neutron dosimetry for biology and medicine. ICRU Report 26 (Bethesda, MD: ICRU) (1977).

13. Currie, L. Limits for quantitative detection and quantitative determination. Anal. Chem. 40(3), 586-593 (1968). 